\title{
A Domain View of Timed Behaviors *
}

\author{
Roman Dubtsov $^{1}$, Elena Oshevskaya ${ }^{2}$, and Irina Virbitskaite ${ }^{2}$ \\ 1 Institute of Informatics System SB RAS, \\ 6, Acad. Lavrentiev av., 630090, Novosibirsk, Russia; \\ 2 Institute of Mathematics SB RAS, \\ 4, Acad. Koptyug av., 630090, Novosibirsk, Russia; \\ dubtsov, eso, virb@iis.nsk.su
}

\begin{abstract}
The intention of this paper is to introduce a timed extension of transition systems with independence, and to study its categorical interrelations with other timed "true-concurrent" models. In particular, we show the existence of a chain of coreflections leading from a category of the model of timed transition systems with independence to a category of a specially defined model of marked Scott domains. As an intermediate semantics we use a model of timed event structures, able to properly capture causality, conflict, and concurrency among events which arise in the presence of time delays of the events.
\end{abstract}

\section{Introduction}

The behaviour of concurrent systems is often specified in terms of states and transitions between states, the labels on the transitions represent the observable part of system's behaviour. The simplest formal model of computation able to express naturally this idea is that of labelled transition systems. However, they are a representative of the interleaving approach to concurrency and hence do not allow one to draw a natural distinction between interleaved and concurrent executions of system's actions. Two most popular "true concurrent" extensions of transition systems, aiming to overcome limitations of the interleaving approach, are asynchronous transition systems, introduced independently by Bednarczyk [1] and Shields [2], and transitions systems with independence, proposed by Winskel and Nielsen [3].

Category theory [4] has been successfully exploited to structure the tangled world of models for concurrency. Within this framework, objects of categories represent processes and morphisms correspond to behavioural relations between the processes, i.e. to simulations. The category-theoretic approach allows for natural formalization of the fact that one model is more expressive than another in terms of an "embedding", most often taking the form of a coreflection, i.e. an adjunction in which the unit is an isomorphism. For example, Hildenbrandt and

\footnotetext{
* The second author is supported in part by the RFBR (grant 12-01-00873-a), by the President Program "Leading Scientific Schools" (grant NSh-7256.2010.1), and by the Federal Program "Research and educational personnel for innovative Russia" (grant $8206)$.
} 
Sassone [5] have constructed a full subcategory of a category of asynchronous transition systems and have shown the existence of a coreflection between the subcategory and a category of transition systems with independence. In their next paper [6], the authors have enriched the model of transition systems with independence by adding multi-arcs and have yielded a precise characterization of the model in terms of (event-maximal, diamond-extensional) labeled asynchronous transition systems, by constructing functors between categories of the models.

It is generally acknowledged that time plays an important role in many concurrent and distributed systems. This has motivated the lifting of the theory of untimed systems to the real-time setting. Timed transition system like models have been studied thoroughly within the two last decades (see [7,8] among others), while timed "true concurrent" extensions have hitherto received scant attention.

The aim of this paper is to introduce a timed extension of transition systems with independence, and to study its categorical interrelations with other timed "true-concurrent" models. In particular, we show the existence of a chain of coreflections leading from a category of the model of timed transition systems with independence to a category of a specially defined model of marked Scott domains. As an intermediate semantics we use a model of timed event structures, able to properly capture causality, concurrency, and conflict among events which arise in the presence of time delays of the events.

The paper is organized as follows. In Section 2, the notions and notations concerning the structure and behaviour of timed transition systems with independence are described. Also, an unfolding of timed transition systems with independence is constructed, and it is shown that together with the inclusion functor the unfolding functor defines a coreflection. Section 3 establishes the interrelations in terms of the existence of a coreflection between timed occurrence transition systems with independence and timed event structures. In Section 4, using the equivalence of the categories of timed event structures and marked Scott domains, stated in [9], functors between the categories of timed transition systems with independence and marked Scott domains are constructed to constitute a coreflection. Section 5 provides a direct translation from timed transition systems with independence to marked Scott domains, established in the categorical setting. In section 6 , we conclude with a short summary of the discovered relationships.

\section{Timed Transition Systems with Independence}

In this section, we first describe the basic notions and notations concerning the structure and behaviour of timed transition systems with independence.

We start with untimed case. A transition system with independence is a tuple $T I=\left(S, s^{I}, L\right.$, Tran, $\left.I\right)$, where $S$ is a countable set of states, $s^{I} \in S$ is the

initial state, $L$ is a countable set of labels, Tran $\subseteq S \times L \times S$ is the transition relation, and $I \subseteq$ Tran $\times$ Tran is the irreflexive, symmetric independence relation, 
such that, using $\prec$ to denote the following relation on transitions $\left(s, a, s^{\prime}\right) \prec$ $\left(s^{\prime \prime}, a, u\right) \Longleftrightarrow \exists\left(s, b, s^{\prime \prime}\right),\left(s^{\prime}, b, u\right) \in \operatorname{Tran}$ s.t. $\left(s, a, s^{\prime}\right) I\left(s, b, s^{\prime \prime}\right) \wedge\left(s, a, s^{\prime}\right) I$ $\left(s^{\prime}, b, u\right) \wedge\left(s, b, s^{\prime \prime}\right) I\left(s^{\prime \prime}, a, u\right)$, and $\sim$ for the least equivalence relation containing $\prec$, we have:

1. $\left(s, a, s^{\prime}\right) \sim\left(s, a, s^{\prime \prime}\right) \Rightarrow s=s^{\prime \prime}$,

2. $\left(s, a, s^{\prime}\right) I\left(s, b, s^{\prime \prime}\right) \Rightarrow \exists\left(s^{\prime}, b, u\right),\left(s^{\prime \prime}, a, u\right) \in \operatorname{Tran} .\left(s, a, s^{\prime}\right) I\left(s^{\prime}, b, u\right) \wedge$ $\left(s, b, s^{\prime \prime}\right) I\left(s^{\prime \prime}, a, u\right)$

3. $\left(s, a, s^{\prime}\right) I\left(s^{\prime}, b, u\right) \Rightarrow \exists\left(s, b, s^{\prime \prime}\right),\left(s^{\prime \prime}, a, u\right) \in \operatorname{Tran} .\left(s, a, s^{\prime}\right) I\left(s, b, s^{\prime \prime}\right) \wedge$ $\left(s, b, s^{\prime \prime}\right) I\left(s^{\prime \prime}, a, u\right)$,

4. $\left(s, a, s^{\prime}\right) \sim\left(s^{\prime \prime}, a, u\right) I\left(w, b, w^{\prime}\right) \Rightarrow\left(s, a, s^{\prime}\right) I\left(w, b, w^{\prime}\right)$.

Let $\operatorname{Diam}_{a, b}\left(s, s^{\prime}, s^{\prime \prime}, u\right) \Longleftrightarrow \exists\left(s, a, s^{\prime}\right),\left(s, b, s^{\prime \prime}\right),\left(s^{\prime}, b, u\right),\left(s^{\prime \prime}, a, u\right) \in$ Tran . $\left(s, a, s^{\prime}\right) I\left(s, b, s^{\prime \prime}\right) \wedge\left(s, a, s^{\prime}\right) I\left(s^{\prime}, b, u\right) \wedge\left(s, b, s^{\prime \prime}\right) I\left(s^{\prime \prime}, a, u\right)$. We say that the transitions above form an independence diamond, and denote the $\sim$-equivalence class of a transition $t \in$ Tran as $[t]$.

A transition system with independence functions by executing transitions from one state to another. A possibly infinite sequence $\pi=t_{0} t_{1} \ldots$ with $t_{i}=$ $\left(s_{i}, a_{i}, s_{i+1}\right) \in \operatorname{Tran}(i \geq 0)$ is called a path. The starting state of $\pi$ is denoted as $\operatorname{dom}(\pi)$, and the ending state as $\operatorname{cod}(\pi)$ if $\pi$ is a finite path. A computation is a path $\pi$ such that $\operatorname{dom}(\pi)=s^{I}$. Let $\operatorname{Comp}(T I)\left(\operatorname{Comp}^{0}(T I)\right)$ be the set of all (finite) computations of $T I$. A transition $t$ is said to be reachable, if there exists a computation $\pi \in \operatorname{Comp}^{0}(T I)$ such that $t$ appears in $\pi$. From now on, we consider only those transition systems with independence in which all transitions are reachable. Let $\simeq \subseteq \operatorname{Comp}(T I) \times \operatorname{Comp}(T I)$ be the least equivalence relation such that $\pi_{s}\left(s, a, s^{\prime}\right)\left(\overline{s^{\prime}}, b, u\right) \pi_{v} \simeq \pi_{s}\left(s, b, s^{\prime \prime}\right)\left(s^{\prime \prime}, a, u\right) \pi_{v} \Longleftrightarrow \operatorname{Diam}_{a, b}\left(s, s^{\prime}, s^{\prime \prime}, u\right)$, and let $[\pi]$ stand for the $\simeq$-equivalence class of a computation $\pi$.

We now incorporate time into the model of transition systems with independence. By analogy with the paper [8], we assume a global, fictitious clock, whose actions advance time by nonuniform amounts and whose value is set to zero at the beginning of system's functioning. All transitions are associated with timing constraints represented as minimal and maximal time delays, and happen "instantaneously", while timing constraints restrict the times at which transitions may be executed. Unlike the paper [8], in our timed model the time domain is changed to the integers, and the maximal delays associated with transitions are always equal to $\infty$, therefore they are not specified explicitly.

Let $\mathbb{N}$ be the set of non-negative integers.

Definition 1. A timed transition system with independence is a tuple TTI= $\left(S, s^{I}, L, T r a n, I, \delta\right)$, where $\llbracket T T I \rrbracket=\left(S, s^{I}, L\right.$, Tran,$\left.I\right)$ is the underlying transition system with independence, and $\delta:$ Tran $\rightarrow \mathbb{N}$ is the delay function such that $\delta(t)=\delta\left(t^{\prime}\right)$ for any $t, t^{\prime} \in$ Tran such that $t \sim t^{\prime}$.

A timed computation of a timed transition system with independence TTI = $\left(S, s^{I}, L, \operatorname{Tran}, I, \delta\right)$ is a pair $\Pi=(\pi, \tau) \in\left(\operatorname{Comp}\left(\left(S, s^{I}, L, \operatorname{Tran}, I\right)\right) \times(\mathbb{N} \cup\right.$ $\{\infty\}))$ with $\tau \geq \delta(\pi)=\sup \{\delta(t) \mid t \in \pi\}$. Define $\operatorname{dom}(\Pi)=\operatorname{dom}(\pi)$ and $\operatorname{cod}(\Pi)=\operatorname{cod}(\pi)$. We denote the set of all (finite) timed computations of TTI as 
$\operatorname{TComp}(T T I)\left(\operatorname{TComp}^{0}(T T I)\right)$, and write $\Pi \simeq_{\tau} \Pi^{\prime}$ iff $\pi \simeq \pi^{\prime}$ and $\tau=\tau^{\prime}$. It is easy to see that $\simeq_{\tau}$ is an equivalence relation; the $\simeq_{\tau^{-} \text {-equivalence class of a timed }}$ computation $\Pi$ is denoted as $[\Pi]_{\tau}$. Let $\operatorname{TComp}_{\simeq_{\tau}}(T T I)\left(\operatorname{TComp}_{\simeq_{\tau}}^{0}(T T I)\right)$ be the sets of $\simeq_{\tau}$-equivalence classes of all (finite) timed computations of $T T I$.

For timed transition systems with independence $T T I=\left(S, s^{I}, L, T r a n, I, \delta\right)$ and $T T I^{\prime}=\left(S^{\prime}, s^{\prime I}, L^{\prime}, T r a n^{\prime}, I^{\prime}, \delta^{\prime}\right)$, a morphism $h: T T I \rightarrow T T I^{\prime}$ is a pair of mappings $h=\left(\sigma: S \rightarrow S^{\prime}, \lambda: L \rightarrow^{*} L^{\prime}\right)^{3}$ such that:

1. $\sigma\left(s^{I}\right)=s^{\prime I}$,

2. $\left(s, a, s^{\prime}\right) \in \operatorname{Tran} \Rightarrow\left(\sigma(s), \alpha(a), \sigma\left(s^{\prime}\right) \in \operatorname{Tran}^{\prime}\right.$ if $a \in \operatorname{dom} \lambda$, and $\sigma(s)=$ $\sigma\left(s^{\prime}\right)$, otherwise,

3. $\left(s, a, s^{\prime}\right) I\left(\bar{s}, \bar{a}, \bar{s}^{\prime}\right)$ and $a, \bar{a} \in \operatorname{dom} \lambda \Rightarrow\left(\sigma(s), \alpha(a), \sigma\left(s^{\prime}\right) I^{\prime}\left(\sigma(\bar{s}), \alpha(\bar{a}), \sigma\left(\bar{s}^{\prime}\right)\right.\right.$,

4. $\delta^{\prime}\left(\left(\sigma(s), \alpha(a), \sigma\left(s^{\prime}\right)\right)\right) \leq \delta\left(\left(s, a, s^{\prime}\right)\right)$.

Timed transition systems with independence and morphisms between them form a category TTSI with unit morphisms $\mathbf{1}_{T T I}=\left(\mathbf{1}_{S}, \mathbf{1}_{L}\right): T T I \rightarrow T T I$ for any $T T I=\left(S, s^{I}, L, \operatorname{Tran}, I, \delta\right)$, and with composition defined in a componentwise manner.

We next aim at unfolding of timed transition systems with independence. To that end, we first define a subclass of timed transition systems with independence that serves as a target of unfolding. After that, we construct an unfolding mapping and show that together with the inclusion functor the unfolding functor defines a coreflection.

Definition 2. $A$ timed occurrence transition system with independenceToTI= $\left(S, s_{0}, L, T r a n, I, \delta\right)$ is an acyclic timed transition system with independence such that $\left(s^{\prime \prime}, a, u\right) \neq\left(s^{\prime}, b, u\right) \in \operatorname{Tran} \Rightarrow \exists s \in S$ s.t. $\operatorname{Diam}_{a, b}\left(s, s^{\prime}, s^{\prime \prime}, u\right)$, for all $\left(s^{\prime \prime}, a, u\right),\left(s^{\prime}, b, u\right) \in$ Tran.

Let ToTSI be the full subcategory of the category TTSI.

Define an unfolding mapping ttsi.totsi : TTSI $\rightarrow$ ToTSI as follows. For a timed transition system with independence $T T I=\left(S, s^{I}, L\right.$, Tran $\left., I, \delta\right)$, specify ttsi.totsi $(T T I)$ as $\left(S_{\simeq_{\tau}},\left[\left(s^{I}, 0\right)\right]_{\tau}, L, \operatorname{Tran}_{\simeq_{\tau}}, I_{\simeq_{\tau}}, \delta_{\simeq_{\tau}}\right)$, where

$-S_{\simeq_{\tau}}=\left\{[\Pi=(\pi, \delta(\pi))]_{\tau} \in \operatorname{TComp}_{\simeq_{\tau}}^{0}(T T I)\right\}$,

$-\left([\Pi=(\pi, \delta(\pi))]_{\tau}, a,\left[\Pi^{\prime}=\left(\pi^{\prime}, \delta\left(\pi^{\prime}\right)\right)\right]_{\tau}\right) \in \operatorname{Tran}_{\simeq_{\tau}} \Longleftrightarrow \exists t_{\pi, \pi^{\prime}}=\left(s, a, s^{\prime}\right) \in$ Tran.$\Pi^{\prime} \simeq_{\tau}\left(\pi t_{\pi, \pi^{\prime}}, \max \left\{\delta(\pi), \delta\left(\pi^{\prime}\right)\right\}\right)$,

$-\left([\Pi]_{\tau}, a,\left[\Pi^{\prime}\right]_{\tau}\right) I_{\simeq_{\tau}}\left([\bar{\Pi}]_{\tau}, b,\left[\bar{\Pi}^{\prime}\right]_{\tau}\right) \Longleftrightarrow t_{\pi, \pi^{\prime}} I t_{\bar{\pi}, \bar{\pi}^{\prime}}$,

$-\delta_{\simeq_{\tau}}\left([\Pi]_{\tau}, a,\left[\Pi^{\prime}\right]_{\tau}\right)=\delta\left(t_{\pi, \pi^{\prime}}\right)$.

Lemma 1. Given a timed transition system with independence TTI, ttsi.totsi(TTI) is a timed occurrence transition system with independence.

\footnotetext{
${ }^{3} \mathrm{~A}$ partial mapping from a set $A$ into a set $B$ is denoted as $f: A \rightarrow^{*} B$. Let $\operatorname{dom} f=\{a \in A \mid f(a)$ is defined $\}$. For a subset $A^{\prime} \subseteq A$, define $f A^{\prime}=\left\{f\left(a^{\prime}\right) \mid a^{\prime} \in\right.$ $\left.A^{\prime} \cap \operatorname{dom} f\right\}$.
} 
In order to demonstrate that the mapping ttsi.totsi is adjoint to the inclusion functor ToTSI $\hookrightarrow$ TTSI, we define a mapping and prove that it is the unit of this adjunction. For a transition system with independence TTI, let $\varepsilon_{T T I}=$ $\left(\sigma_{\varepsilon}, 1_{L}\right):$ ttsi.totsi $(T T I) \rightarrow T T I$, where $\sigma_{\varepsilon}\left([\Pi]_{\tau}\right)=\operatorname{cod}(\Pi)$ for all $[\Pi]_{\tau} \in S_{\simeq_{\tau}}$. It is easy to see that $\varepsilon_{T T I}$ is a morphism of TTSI.

Lemma 2 ( $\varepsilon_{T T I}$ is couniversal). For any object TTI of TTSI, any object ToTI of ToTSI and any morphism $h:$ ToTI $\rightarrow$ TTI of TTSI, there exists a unique morphism $h^{\prime}:$ ToTI $\rightarrow$ ttsi.totsi(TTI) of ToTSI such that $h=\varepsilon_{T T I} \circ h^{\prime}$.

The next theorem presents a categorical characterization of the unfolding.

Theorem 1 ( $\hookrightarrow \dashv$ ttsi.totsi). The unfolding mapping ttsi.totsi extends to a functor from TTSI $\rightarrow$ ToTSI which is right adjoint to the functor $\hookrightarrow:$ ToTSI $\rightarrow$ TTSI. Moreover, this adjunction is a coreflection.

\section{Timed Event Structures}

In this section we relate timed occurrence transition systems with independence and timed event structures, establishing the close relationships between categories of the models.

We start with the definition of an untimed variant of event structures. An event structure is a triple $\mathcal{E}=(E, \leq, \#)$, where $E$ is a countable set of events; $\leq \subseteq E \times E$ is a partial order (the causality relation) such that $\downarrow e=\left\{e^{\prime} \in E \mid\right.$ $\left.e^{\prime} \leq e\right\}$ is a finite set for each $e \in E, \# \subseteq E \times E$ is the symmetric irreflexive conflict relation such that $e \# e^{\prime} \leq e^{\prime \prime} \Rightarrow e \# e^{\prime \prime}$. A set of events $C \subseteq E$ is said to be a configuration of an event structure $\mathcal{E}$ if $\forall e \in C$. $\downarrow e \subseteq C$, and $\forall e, e^{\prime} \in C . \neg\left(e \# e^{\prime}\right)$. We say that events $e, e^{\prime} \in E$ are concurrent and write $e \smile e^{\prime}$ if $\neg\left(e \leq e^{\prime} \vee e^{\prime} \leq e^{\prime} \vee e \# e^{\prime}\right)$. Introduce the concept of a reflexive conflict as follows: $e \mathbb{W} e^{\prime} \Longleftrightarrow e \# e^{\prime} \vee e=e^{\prime}$.

We now recall the definition of timed event structures from [9]. Similarly to the model of timed transition systems with independence, there is a global nonnegative integer-valued clock. Each event in the structure is associated with a time delay with respect to the initial time moment; i.e., if an event $e$ is associated with a time delay $t$, then $e$ may not occur earlier than all the predecessors of the event occur and the clock shows time $t$. In this case, the event itself occurs instantaneously.

Definition 3. A timed event structure is a tuple $\mathcal{T} \mathcal{E}=(E, \leq, \#, \Delta)$, where $(E, \leq, \#)$ is an event structure and $\Delta: E \rightarrow \mathbb{N}$ is the delay function such that $e^{\prime} \leq e \Rightarrow \Delta\left(e^{\prime}\right) \leq \Delta(e)$.

A timed configuration of $\mathcal{T E}$ is a pair $(C, \tau)$, where $C$ is a configuration of $(E, \leq, \#)$ and $\tau \in \mathbb{N} \cup\{\infty\}$ such that $\tau \geq \Delta(C)=\sup \{\Delta(e) \mid e \in C\}$. The set of all (finite) timed configurations of a timed event structure $\mathcal{T} \mathcal{E}$ is denoted as $\operatorname{TConf}(\mathcal{T E})\left(\operatorname{TConf}^{0}(\mathcal{T E})\right)$. We define a transition relation $\longrightarrow$ on the set 
$\operatorname{TConf}(\mathcal{T E})$ as follows: $(C, t) \longrightarrow\left(C^{\prime}, t^{\prime}\right)$ if $C \subseteq C^{\prime}$ and $t \leq t^{\prime}$. Clearly, the relation $\longrightarrow$ specifies a partial order on the set $\operatorname{TConf}(\mathcal{T E})$.

Let $\mathcal{T E}=(E, \leq, \#, \Delta)$ and $\mathcal{T} \mathcal{E}^{\prime}=\left(E^{\prime}, \leq^{\prime}, \#^{\prime}, \Delta^{\prime}\right)$ be timed event structures. A partial mapping $\theta: E \rightarrow^{*} E^{\prime}$ is a morphism if $\downarrow \theta(e) \subseteq \theta \downarrow e ; \theta(e) W \theta\left(e^{\prime}\right) \Rightarrow$ $e W e^{\prime}$, for all $e, e^{\prime} \in \operatorname{dom} \theta ; \Delta^{\prime}(\theta(e)) \leq \Delta(e)$, for all $e \in \operatorname{dom} \theta$. Timed event structures with their morphisms define a category TES with unit morphisms $\mathbf{1}_{T S}=\mathbf{1}_{E}: T S \rightarrow T S$ for all $T S=(E, \leq, \#, \Delta)$ and the composition being a usual composition of partial functions.

We now establish the relationships between the categories of timed event structures and timed occurrence transition systems with independence. For this purpose, we first define a mapping tpes.totsi : TPES $\rightarrow$ ToTSI extending the mapping pes.otsi from [3] to the timed case. For a timed event structure $\mathcal{T E}=$ $(E, \leq, \#, \Delta)$, let tpes.totsi $(\mathcal{T E})$ be $\left(S_{\mathcal{T E}}, s_{\mathcal{T} \mathcal{E}}^{I}, L_{\mathcal{T} \mathcal{E}}, \operatorname{Tran}_{\mathcal{T E}}, I_{\mathcal{T} \mathcal{E}}, \delta_{\mathcal{T} \mathcal{E}}\right)$, where

$-S_{\mathcal{T E}}=\left\{(C, \Delta(C)) \in \operatorname{TConf}^{0}(\mathcal{T E})\right\}$

$-s_{\mathcal{T E}}^{I}=(\varnothing, 0)$

$-L_{\mathcal{T E}}=E$;

- $\left((C, \Delta(C)), e,\left(C^{\prime}, \Delta\left(C^{\prime}\right)\right)\right) \in \operatorname{Tran}_{\mathcal{T E}} \Longleftrightarrow C^{\prime} \backslash C=\{e\} ;$

- $\left((C, \Delta(C)), e,\left(C^{\prime}, \Delta\left(C^{\prime}\right)\right)\right) I_{\mathcal{T} \mathcal{E}}\left((\bar{C}, \Delta(\bar{C})), \bar{e},\left(\bar{C}^{\prime}, \Delta\left(\bar{C}^{\prime}\right)\right)\right) \Longleftrightarrow e \smile \bar{e} ;$

$-\delta_{\mathcal{T} \mathcal{E}}\left((C, \Delta(C)), e,\left(C^{\prime}, \Delta\left(C^{\prime}\right)\right)\right)=\Delta(e)$.

It is easy to see that the above definition is correct, i.e. tpes.totsi maps timed event structures to timed occurrence transition systems with independence.

Next, we construct a mapping totsi.tpes : ToTSI $\rightarrow$ TPES. For a timed occurrence transition system with independence $T o T I=\left(S, s^{I}, L, \operatorname{Tran}, I, \delta\right)$, let totsi.tpes(ToTI) be $\left(\operatorname{Tran}_{\sim}, \leq, \#, \Delta\right)$, where

$-\operatorname{Tran}_{\sim}=\{[t] \mid t \in \operatorname{Tran}\}$,

$-[t]<\left[t^{\prime}\right] \Longleftrightarrow$

$\forall\left(\pi \bar{t}^{\prime}, \tau\right) \in \operatorname{TComp}^{0}($ ToTI $) . \bar{t}^{\prime} \sim t^{\prime} \Rightarrow(\exists \bar{t} \in \pi . \bar{t} \sim t) ; \leq=<\cup=$,

$-[t] \#\left[t^{\prime}\right] \Longleftrightarrow$

$\forall(\pi, \tau) \in \operatorname{TComp}^{0}(T o T I), \forall \bar{t} \in[t], \forall \bar{t}^{\prime} \in\left[t^{\prime}\right] . \bar{t} \in \pi \Rightarrow \bar{t}^{\prime} \notin \pi$,

$-\Delta([t])=\max \left\{\delta\left(t^{\prime}\right) \mid\left[t^{\prime}\right] \leq[t]\right\}$.

On morphisms $h=(\sigma, \lambda):$ ToTI $\rightarrow$ ToTI' in ToTSI, the mapping totsi.tpes acts as follows: totsi.tpes $(h)=\theta$, where $\theta\left(\left[\left(s, a, s^{\prime}\right)\right]\right)=\left[\left(\sigma(s), \lambda(a), \sigma\left(s^{\prime}\right)\right]\right.$, if $a \in \operatorname{dom} \lambda$, and $\theta\left(\left[\left(s, a, s^{\prime}\right)\right]\right)$ is undefined, otherwise.

Proposition 1. totsi.tpes: ToTSI $\rightarrow$ TPES is a functor.

Finally, we define the unit of the adjunction. For a timed event structure $\mathcal{T E}$, let $\eta_{\mathcal{T E}}: E_{\mathcal{T E}} \rightarrow E_{\text {totsi.tpesotpes.totsi }(\mathcal{T E})}$ be a mapping such that $\eta_{\mathcal{T} \mathcal{E}}(e)=$ $[(C, \Delta(C)), e,(C \cup\{e\}, \Delta(C \cup\{e\}))]$. It is straightforward to show that $\eta_{\mathcal{T E}}$ is an isomorphism in TPES. In order to demonstrate the existence of the adjunction, we need to check that $\eta_{\mathcal{T E}}$ is indeed a unit, i.e. it is universal.

Lemma 3 ( $\eta_{\mathcal{T} \mathcal{E}}$ is universal).

For any object $\mathcal{T E}$ of TPES, any object ToTI of ToTSI, and any morphism $\theta: \mathcal{T E} \rightarrow$ totsi.tpes(ToTI) in TPES, there exists a unique morphism $h:$ tpes.totsi $(\mathcal{T E}) \rightarrow$ ToTI in ToTSI such that $\theta=$ totsi.tpes $(h) \circ \eta_{\mathcal{T} \mathcal{E}}$. 
The next theorem establishes the existence of a coreflection between the categories of timed event structures and timed occurrence transition systems with independence.

Theorem 2 (tpes.totsi $\dashv$ totsi.tpes). The map tpes.totsi can be extended to a functor tpes.totsi : TPES $\rightarrow$ ToTSI, which is left adjoint to the functor totsi.tpes. Moreover, this adjunction is a coreflection.

\section{Marked Scott Domains}

In this section, we extend the established chain of coreflections to marked Scott domains. To that end, we first recall related notions and notations.

Let $(D, \sqsubseteq)$ be a partial order, $d \in D$ and $X \subseteq D$. Then,

$-\uparrow d=\left\{d^{\prime} \in D \mid d \sqsubseteq d^{\prime}\right\}$ is an upper cone of element $d, \downarrow d=\left\{d^{\prime} \in D \mid d^{\prime} \sqsubseteq d\right\}$ is a lower cone of element $d$,

- $X$ is downward (upward) closed if $\downarrow d \subseteq X$ ( $\uparrow d \subseteq X$ ) for every $d \in X$,

- $X$ is a compatible set (denoted as $X \uparrow$ ), if the following assertion is true: $\exists d \in D \forall x \in X . x \sqsubseteq d$, i.e., $X$ has an upper bound. If $X=\{x, y\}$, we write $x \uparrow y$ instead of $\{x, y\} \uparrow$. The least upper bound of the set $X$ is denoted as $\bigsqcup X$ (if it exists), and the greatest lower bound is denoted as $\prod X$ (if it exists). The least upper bound of two elements $x$ and $y$ is denoted as $x \sqcup y$, and the greatest lower bound, as $x \sqcap y$.

- $X$ is a finitely compatible set if any finite subset of it $X^{\prime} \subseteq X$ is compatible.

$-X$ is a (upper) directed set if any finite subset of it $X^{\prime} \subseteq X$ has an upper bound belonging to the set $X$ (thus, $X$ is a finitely compatible and nonempty set).

$-(D, \sqsubseteq)$ is a directed-complete partial order (dcpo for short) if every directed subset $X \subseteq D$ has $\bigsqcup X$.

$-d$ is a finite (compact) element of a dcpo $(D, \sqsubseteq)$ if, for any directed subset $X \subseteq D$, the following assertion is true: $d \sqsubseteq \bigsqcup X \Rightarrow \exists x \in X . d \sqsubseteq x$. The set of finite elements is denoted as $C(D)$.

- A dcpo $(D, \sqsubseteq)$ is said to be algebraic if, for any $d \in D, d=\bigsqcup\{e \sqsubseteq d \mid e \in$ $C(D)\}$. It is said to be $\omega$-algebraic if $C(D)$ is countable.

- $(D, \sqsubseteq)$ is a consistently complete partial order (ccpo) if any finitely compatible subset $X \subseteq D$ has $\bigsqcup X$. Clearly, a ccpo has the least element $\perp=\bigsqcup \emptyset$, and is also a depo.

- An $\omega$-algebraic ccpo is called a Scott domain. A Scott domain $(D, \sqsubseteq)$ is said to be finitary if $\downarrow d$ is finite for every $d \in C(D)$.

Describe some properties of Scott domains. An element $p$ of a Scott domain $(D, \sqsubseteq)$ is said to be prime if, for any compatible subset $X \subseteq D . p \sqsubseteq \bigsqcup X \Rightarrow \exists x \in$ $X . p \sqsubseteq x$. The set of the prime elements is denoted as $P(D)$. A Scott domain $(D, \sqsubseteq)$ is called prime algebraic if, for any $d \in D, d=\bigsqcup\{p \sqsubseteq d \mid p \in P(D)\}$ and coherent if all subsets $X \subseteq D$ satisfying the condition $\forall d^{\prime}, d^{\prime \prime} \in X . d^{\prime} \uparrow d^{\prime \prime}$ have $\bigsqcup X$. 
Let $(D, \sqsubseteq)$ be a Scott domain and $\prec=\sqsubset \backslash \sqsubset^{2}$ be a covering relation. For elements $d, d^{\prime} \in D$ such that $d \prec d^{\prime}$, the pair $\left[d, d^{\prime}\right]$ is called a prime interval. The set of all prime intervals is denoted as $I(D)$. We write $\left[c, c^{\prime}\right] \leq\left[d, d^{\prime}\right]$ if and only if $c=c^{\prime} \sqcap d \vee d^{\prime}=c^{\prime} \sqcup d$. The relation $\sim$ is defined to be a transitive symmetric closure of the relation $\leq$. Note that $\sim$-equivalent prime intervals model one and the same action. Let $\left[d, d^{\prime}\right] \sim$ denote the $\sim$-equivalence class of the prime interval $\left[d, d^{\prime}\right]$.

Now we are ready to present the definition of marked Scott domains. Informally, a marked Scott domain is meant to be a prime algebraic, finitary, and coherent Scott domain with the prime intervals modeling two - instantaneous and delayed - types of system actions. The former actions do not require time and are marked by zero, and the latter take one unit of time and are marked by one. It is natural to require that the $\sim$-equivalent prime intervals corresponding to one and the same system action are marked identically.

Definition 4. A marked domain is a triple $(D, \sqsubseteq, m)$, where $(D, \sqsubseteq)$ is a prime algebraic, finitary, and coherent Scott domain and $m: I(D) \longrightarrow\{0,1\}$ is a marking such that $\left[c, c^{\prime}\right] \sim\left[d, d^{\prime}\right] \Rightarrow m\left(\left[c, c^{\prime}\right]\right)=m\left(\left[d, d^{\prime}\right]\right)$.

Introduce auxiliary notions and notations. For $d, d^{\prime} \in D$ and $i \in\{0,1\}$, we write $d \prec^{i} d^{\prime}$, if $d \prec d^{\prime} \wedge m\left(\left[d, d^{\prime}\right]\right)=i$, and $d \preccurlyeq^{i} d^{\prime}$, if $d \prec^{i} d^{\prime} \vee d=d^{\prime}$; $\sqsubseteq^{i}=\left(\prec^{i}\right)^{*} ; \downarrow^{i} d=\left\{d^{\prime} \mid d^{\prime} \sqsubseteq^{i} d\right\}$, and $\uparrow^{i} d=\left\{d^{\prime} \mid d \sqsubseteq^{i} d^{\prime}\right\} ; P^{i}(D)=\{p \in$ $P(D) \mid \exists d \in D . m([d, p])=i\}$. For a finite element $d \in D$ and a covering chain $\sigma$ having the form $\perp=d_{0} \prec^{k_{1}} d_{1} \cdots d_{n-1} \prec^{k_{n}} d_{n}=d$ (the chain is finite as $(D, \sqsubseteq)$ is finitary), define the norm of $d$ along $\sigma$ by $\|d\|_{\sigma}=\sum_{i=1}^{n} k_{i}$. Since $(D, \sqsubseteq)$ is a prime algebraic Scott domain and $m$ respects $\sim$, the value of $\|d\|_{\sigma}$ does not depend on $\sigma$. Therefore, we shall use $\|d\|$ to denote the norm of a finite element $d$. For a non-finite element $d \in D$, its norm is defined as follows: $\|d\|=\sup \left\{\left\|d^{\prime}\right\| \mid d^{\prime} \in \downarrow d \cap C(D)\right\}$. A marked domain $(D, \sqsubseteq, m)$ is said to be linear if for any $d \in D$ such that $\|d\|<\infty,\left(\uparrow^{1} d, \sqsubseteq^{1}\right) \cong(\mathbb{N}, \leq)$; regular if for any $d, d^{\prime} \in D, d \uparrow d^{\prime} \Rightarrow \forall d_{1} \in \uparrow^{1} d, \forall d_{1}^{\prime} \in \uparrow^{1} d^{\prime} .\left(d_{1} \uparrow d_{1}^{\prime}\right)$.

It is not difficult to see that linear regular marked domains, together with the additive stable mappings [10] preserving $\preccurlyeq^{0}$ and $\prec^{1}$, form the category MDom.

As shown in [9], marked Scott domains are related with timed event structures via a pair of functors tpes.mdom : TPES $\rightarrow$ MDom and mdom.tpes : MDom $\rightarrow$ TPES defined as follows ${ }^{4}$.

For a timed event structure $\mathcal{T E}=(E, \leq, \#, \Delta)$, let tpes.mdom $(\mathcal{T E})$ be $\left(\operatorname{TConf}(\mathcal{T E}), \longrightarrow, m_{\mathcal{T} \mathcal{E}}\right)$, where

$$
m\left(\left[(C, \tau),\left(C^{\prime}, \tau^{\prime}\right)\right]\right)=\left\{\begin{array}{l}
0, \text { if } C^{\prime} \backslash C=\{e\} \wedge \tau^{\prime}=\tau \\
1, \text { if } C^{\prime}=C \wedge \tau^{\prime}=\tau+1
\end{array}\right.
$$

For a marked Scott domain $M D=(D, \sqsubseteq, m) \in$ MDom, define mdom.tpes $(M D)$ to be $(E, \leq, \#, \Delta)$, where $E=P^{0}(D), p \leq p^{\prime} \Longleftrightarrow p \sqsubseteq p$, $p \# p^{\prime} \Longleftrightarrow p \vee p^{\prime}$, and $\Delta(p)=\|p\|$.

\footnotetext{
${ }^{4}$ We do not specify how tpes.mdom and mdom.tpes act on morphisms since it is not essential to this paper.
} 
Theorem 3. [9]. The functors tpes.mdom and mdom.tpes constitute an equivalence between the categories TPES and MDom.

Theorems 1, 2 and 3 yield the following corollary.

Theorem 4. The functor $\hookrightarrow$ otpes.totsi o mdom.tpes : MDom $\rightarrow$ TTSI is left adjoint to the functor tpes.mdom $\circ$ totsi.tpes $\circ$ ttsi.totsi : TTSI $\rightarrow$ MDom. Moreover, this adjunction is a coreflection.

\section{Direct Characterization}

In this section, we establish some relationships between timed transition systems with independence and marked Scott domains in a direct way.

We start with introducing auxiliary notations. For a transition system with independence $T I=\left(S, s^{I}, L, \operatorname{Tran}, I\right)$ and computations $\pi, \pi^{\prime} \in \operatorname{Comp}^{0}(T I)$, we write $\pi \unlhd \pi^{\prime}$ iff there exists a path $\pi^{\prime \prime}$ such that $\pi \pi^{\prime \prime} \simeq \pi^{\prime}$ :

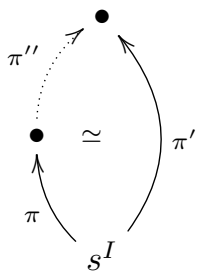

For possibly infinite computations $\pi, \pi^{\prime} \in \operatorname{Comp}(T I)$, let $\pi \unlhd \pi^{\prime}$ iff for every finite prefix $\bar{\pi}$ of $\pi$ there exists a finite prefix $\bar{\pi}^{\prime}$ of $\pi^{\prime}$ such that $\bar{\pi} \unlhd \bar{\pi}$. It is straightforward to check that $\unlhd$ is a partial order on $\operatorname{Comp}(T I)$. Specify a partial order on timed computations as follows: $\Pi=(\pi, \tau) \unlhd_{\tau} \Pi^{\prime}=\left(\pi^{\prime}, \tau^{\prime}\right)$ iff

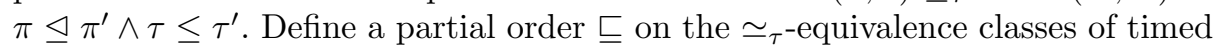
computations as follows: $[\Pi]_{\tau} \sqsubseteq\left[\Pi^{\prime}\right]_{\tau}$ iff $\Pi \unlhd_{\tau} \Pi^{\prime}$.

Lemma 4. $\left(\mathrm{TComp}_{\simeq_{\tau}}(T T I)\right.$, 巨) is a finitary $\omega$-algebraic dcpo. Moreover,

$$
C\left(\left(\mathrm{TComp}_{\simeq_{\tau}}(T T I), \sqsubseteq\right)\right)=\mathrm{TComp}_{\simeq_{\tau}}^{0}(T T I) .
$$

In order to directly relate timed transition systems with independence and marked Scott domains, we construct a mapping ttsi.mdom ${ }^{\prime}:$ TTSI $\rightarrow$ MDom. Before doing so, consider a prime interval $\left[[\Pi=(\pi, \tau)]_{\tau},\left[\Pi^{\prime}=\left(\pi^{\prime}, \tau^{\prime}\right)\right]_{\tau}\right]$ in $\left(\mathrm{TComp}_{\simeq_{\tau}}(T T I), \sqsubseteq\right)$. It is not difficult to check that either $\pi^{\prime} \simeq \pi \wedge \tau^{\prime}=\tau+1$ or $\pi^{\prime} \simeq \pi t \wedge \tau^{\prime}=\tau$ for some transition $t$. Define a map $m_{T T I}: I\left(\left(\mathrm{TComp}_{\simeq_{\tau}}(T T I)\right.\right.$, $\sqsubseteq)) \rightarrow\{0,1\}$ as follows:

$$
m_{T T I}\left(\left[[\Pi]_{\tau},\left[\Pi^{\prime}\right]_{\tau}\right]\right)=\left\{\begin{array}{l}
0, \text { if } \tau=\tau^{\prime} \\
1, \text { otherwise. }
\end{array}\right.
$$

Let ttsi.mdom $(T T I)=\left(\mathrm{TComp}_{\simeq_{\tau}}(T T I), \sqsubseteq, m_{T T I}\right)$, for any timed transition system with independence $T T I$. 
Proposition 2. ttsi.mdom ${ }^{\prime}$ can be extended to a functor ttsi.mdom ${ }^{\prime}$ : TTSI $\rightarrow$ MDom isomorphic to ttsi.mdom $=$ tpes.mdom $\circ$ ottsi.tpes $\circ$ ttsi.ottsi.

At last, we are ready to state the fact which is the last main result of this paper and that provides a direct characterisation.

Theorem 5. ttsi.mdom ${ }^{\prime}$ is right adjoint to mdom.ttsi $=$ tpes.mdomo ottsi.tpes $\circ$ ttsi.ottsi. Moreover, this adjunction is a coreflection.

\section{Conclusion}

We have defined and studied a timed extension of a well-known "true concurrent" model of transition systems with independence and have shown that there exists a chain of coreflections between a category of the model and a category of marked Scott domains as well as a direct translation. The diagram below summarises the established relationships:

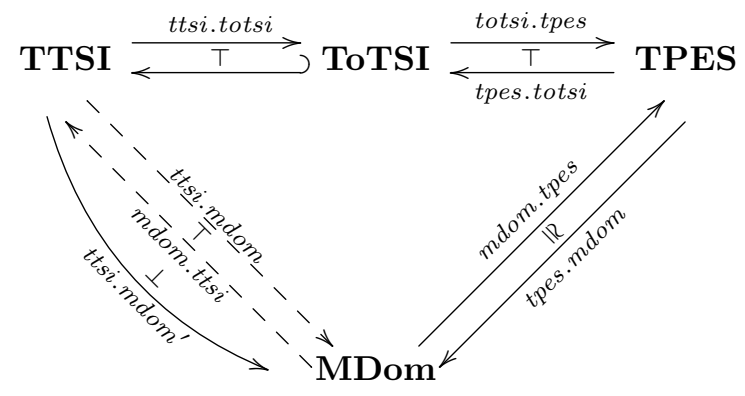

\section{References}

1. Bednarczyk, M.: Categories of asynchronous systems. PhD thesis, University of Sussex, UK (1987)

2. Shields, M.: Concurrent Machines. The Computer Journal 28(5) (1985) 449-465

3. Sassone, V., Nielsen, M., Winskel, G.: Models for concurrency: towards a classification. Theoretical Computer Science 170(1-2) (1996) 297-348

4. McLane, S.: Categories for the working mathematician. Graduate Texts in Mathematics. Springer, Berlin (1971)

5. Hildebrandt, T., Sassone, V.: Comparing Transition Systems with Independence and Asynchronous Transition Systems. International Conference on Concurrency Theory (1996) 84-97

6. Hildebrandt, T., Sassone, V.: Transition Systems with Independence and MultiArcs. BRICS Report Series RS-97-10, BRICS, Department of Computer Science, University of Aarhus, April (1997)

7. Alur, R., Dill, D.: A theory of timed automat. Theoretical computer science 126(2) (1994) 183-235

8. Henzinger, T., Manna, Z., Pnueli, A.: Timed transition systems. In: Real-Time: Theory in Practice, Springer (1992) 226-251 
9. Virbitskaite, I.B., Dubtsov, R.S.: Semantic domains of timed event structures. Programming and Computer Software 34(3) (2008) 125-137

10. Winskel, G.: Event structures. Lecture Notes in Computer Science 255 (1987) 325-392

\section{Appendix A: Elements of Category Theory}

Here we briefly recall notions from category theory [4] important to this paper. Let $G: \mathbf{B} \rightarrow \mathbf{A}$ be a functor between categories $\mathbf{A}$ and $\mathbf{B}$, and let, for each object $A$ of $\mathbf{A}$, there exist an object $F(A)$ of $\mathbf{B}$ and a morphism $\eta_{A}: A \rightarrow G \circ F(A)$ in A that is universal in the following sense: for any morphism $h: A \rightarrow G(B)$ in $\mathbf{A}$, where $B$ is an object of $\mathbf{B}$, there exists a unique morphism $h^{\prime}: F(A) \rightarrow B$ in $\mathbf{B}$ such that $G\left(h^{\prime}\right) \circ \eta_{A}=h$; i.e., the following diagram commutes.

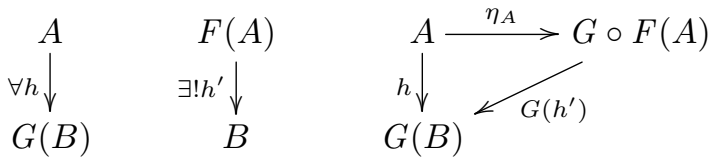

In this case, we say that there exists an adjunction from $\mathbf{A}$ to $\mathbf{B}$, and the family of morphisms $\left\{\eta_{A} \mid A \in \mathbf{A}\right\}$ is said to be a unit of this adjunction. Then, $F$ can be extended to a functor by assuming that, for any morphism $h: A \rightarrow A^{\prime}$ in $\mathbf{A}$, $F(h): F(A) \rightarrow F\left(A^{\prime}\right)$ is a unique morphism in $\mathbf{B}$ such that $G \circ F(h) \circ \eta_{A}=\eta_{A^{\prime}} \circ h$. In this case, $F$ is said to be left adjoint to $G$ (denoted as $F \vdash G$ ), and $G$ right adjoint to $F$ (denoted as $G \dashv F$ ). In addition, if $\eta_{A}$ is an isomorphism for each $A$, then the adjunction is called a coreflection. Categories $\mathbf{A}$ and $\mathbf{B}$ are equivalent if $F$ is adjoint to $G$ and both the unit and counit of the adjunction are isomorphisms. 